\title{
3D finite element simulation of effects of deflection rate on energy absorption for TRIP steel
}

\author{
Asuka Hayashi ${ }^{1}$, Hang Pham ${ }^{1,3}$, and Takeshi Iwamoto ${ }^{2}$ \\ ${ }^{1}$ Graduate School of Engineering, Hiroshima University, 1-4-1 Kagamiyama, Higashi-Hiroshima, Hiroshima 739-8527, \\ Japan \\ 2 Institute of Engineering, Hiroshima University, 1-4-1 Kagamiyama, Higashi-Hiroshima, Hiroshima 739-8527, Japan \\ ${ }^{3}$ Faculty of Engineering, Vietnam National University of Agriculture, Trauquy, Gialam, Hanoi 131004, Vietnam
}

\begin{abstract}
Recently, with the requirement of lighter weight and more safety for a design of automobile, energy absorption capability of structural materials has become important. TRIP (Transformation-induced Plasticity) steel is expected to apply to safety members because of excellent energy absorption capability and ductility. Past studies proved that such excellent characteristics in TRIP steel are dominated by strain-induced martensitic transformation (SIMT) during plastic deformation. Because SIMT strongly depends on deformation rate and temperature, an investigation of the effects of deformation rate and temperature on energy absorption in TRIP is essential. Although energy absorption capability of material can be estimated by $J$-integral experimentally by using pre-cracked specimen, it is difficult to determine volume fraction of martensite and temperature rise during the crack extension. In addition, their effects on $J$-integral, especially at high deformation rate in experiment might be quite hard. Thus, a computational prediction needs to be performed. In this study, bending deformation behavior of pre-cracked specimen until the onset point of crack extension are predicted by 3D finite element simulation based on the transformation kinetics model proposed by Iwamoto et al. (1998). It is challenged to take effects of temperature, volume fraction of martensite and deformation rate into account. Then, the mechanism for higher energy absorption characteristic will be discussed.
\end{abstract}

\section{Introduction}

Recently, a decrease in weight and improvement of safety for automobiles are challenging tasks for automobile industry [1]. Thus, energy-absorption capacity of structural materials has become important to protect the passengers from a high-speed crash of automobiles [2]. From the perspective of safety, nowadays, TRIP (transformation induced plasticity) steel is commonly applied for such structures and components [2] because of the high strength and excellent ductility due to strain-induced martensitic transformation (SIMT) [3]. In addition, TRIP steel might have high energy-absorption capacity because huge amount of kinetic energy might be dissipated during phase transformation process [4]. On the other hand, deformation mode of safety members during crash includes not only axial tension but also bending deformation. Therefore, an investigation of effect of deflection rate on energy-absorption characteristic during bending deformation process for TRIP steel is necessary.

Although many past research works have focused on TRIP steel [5-7], the effect of SIMT on energyabsorption has still insufficiently investigated. Energyabsorption capacity of TRIP steel can be estimated by $J$-integral derived by Rice [8] experimentally by using pre-cracked specimen [9]. However, SIMT process itself is considered quite complicate and it is difficult to determine volume fraction of martensite and temperature rise during the crack extension of tested specimens. Additionally, their effects on energy-absorption characteristic, especially at a high deformation rate might be quite hard to observe in experiment. Thus, a computational prediction needs to be performed.

With the purpose of predicting and controlling the mechanical properties of TRIP steel, Olson and Cohen [10] established a model for SIMT kinetics which can express the temperature dependent of SIMT. After that, Stringfellow et al. [11] generalized the model of Olson and Cohen by including the effect of stress state into the driving force of the martensitic transformation. Then, Tomita and Iwamoto [12] incorporated the effects of the strain rate sensitivity into the model proposed by Stringfellow et al. Iwamoto et al. [13] incorporate the stress state dependence based on the model proposed by Olson and Cohen [10], and Stringfellow [11]. The validity of the model is confirmed by some furthermore researches $[12,13]$.

In this study, bending deformation behaviour of precracked specimen is investigated by $3 \mathrm{D}$ finite element simulation based on the transformation kinetics model proposed by Iwamoto et al. [14]. After confirming the validity of computation, effects of deflection rate, temperature, volume fraction of martensite on energy absorption of TRIP steel until the onset point of crack extension of specimen are examined. Then, the mechanism for energy absorption characteristic in TRIP steel will be discussed. 


\section{Methodology}

\subsection{The constitutive model for TRIP steel}

The rate of the volume fraction of martensite, $f^{\alpha^{\prime}}$ is calculated as shown in the following equation [14].

$$
\begin{gathered}
\dot{f}^{\alpha^{\prime}}=\left(1-f^{\alpha^{\prime}}\right)\left(A \dot{\bar{\varepsilon}}_{(\gamma)}^{\text {sslip }}+B \dot{g}\right) \\
A=\alpha \beta\left(f^{s b}\right)^{n-1}\left(1-f^{s b}\right) \\
B=\eta n_{v}(g)\left(f^{s b}\right)^{n} H(\dot{g}) \\
\alpha=\left(\alpha_{1} T^{2}+\alpha_{2} T+\alpha_{3}-\alpha_{4} \Sigma\right)\left[\frac{\dot{\bar{\varepsilon}}_{(\gamma)}^{p s l i p}}{\dot{\varepsilon}_{y}}\right]^{M} \\
\beta=\frac{\eta}{\sqrt{2 \pi \sigma_{g}}} \int_{-\infty}^{g} \exp \left\{-\frac{\left(g-g_{0}\right)^{2}}{2 \sigma_{g}^{2}}\right\} d g^{\prime} \\
\eta=\frac{K \bar{v}^{\alpha^{\prime}}}{\bar{v}^{s b}}, g=-T+g_{1} \Sigma, \Sigma=\sigma_{\ddot{u}} / 3 \bar{\sigma}
\end{gathered}
$$

where $\dot{\bar{\varepsilon}}_{(\gamma)}^{\text {pslip }}$ is equivalent plastic strain rate by slip deformation in the austenite, $f^{s b}$ is the volume fraction of the shear band, $g$ is driving force for SIMT and $T$ is the absolute temperature. $\Sigma$ is the stress triaxiality parameter, $M$ is the strain-rate sensitivity exponent, $\dot{\varepsilon}_{y}$ is the reference strain rate and $\alpha_{1}-\alpha_{4}$ are material parameters.

To express the deformation mode dependence of the deformation behaviour of TRIP steels, the thermo-elastoviscoplastic constitutive equation for large deformation including transformation strain rate can be formulated as [15]

$$
\begin{gathered}
\check{S}_{i j}=D_{i j k l}^{v} d_{k l}-B_{i j}^{e} \dot{T}-P_{i j} \Theta_{1}-\left(Q_{i j}-\sigma_{i j}\right) \Delta v \dot{f}^{\alpha^{\prime}} \\
D_{i j k l}^{v}=D_{i j k l}^{e}-\Theta_{2} P_{i j} P_{k l}, B_{i j}^{e}=\frac{E}{1-2 v} \alpha_{T} \delta_{i j} \\
P_{i j}=\frac{3 E}{2 \bar{\sigma}(1+v)} \frac{\partial f}{\partial \sigma_{i j}}, Q_{i j}=-P_{i j} \Sigma+\frac{1}{3} \delta_{i j} \frac{E}{1-2 v}
\end{gathered}
$$

where $\breve{S}_{i j}$ is Jaumann rate of Kirchhoff stress, $D_{i j k l}^{e}$ is the elastic stiffness tensor, $\alpha_{T}$ is the thermal expansion coefficient, $E$ is the Young's modulus and $v$ is the Poisson's ratio. According to Tomita and Iwamoto [12], the heat conduction equation accounting for the latent heat due to SIMT $l_{\alpha^{\prime \prime}}$ is can be delivered as

$$
\rho C_{v} \dot{T}=\zeta \sigma_{i j} \dot{\varepsilon}_{i j}^{p}+\kappa^{t} \nabla^{2} T-\rho l_{\alpha^{\prime}} \dot{f}^{\alpha^{\prime}}
$$

where $C_{v}$ is the specific heat at constant volume, $\rho$ is the mess density and $\zeta$ is Taylor-Quinney coefficient.

\subsection{Finite element equations}

In impact condition, inertia force is considered and added into finite element equation as following,

$$
M \ddot{d}^{e}+K \dot{d}^{e}=\dot{f}_{1}+\dot{f}_{2}+\dot{f}_{3}+\dot{f}_{t}+\dot{f}_{g}
$$

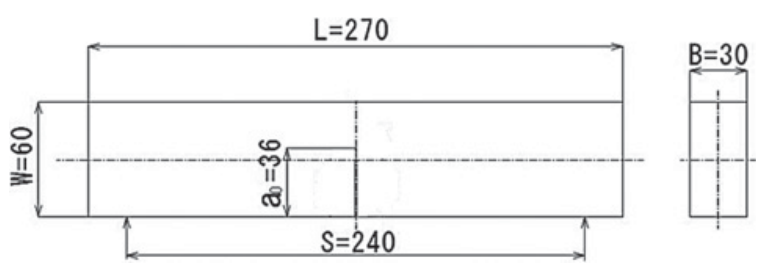

Figure 1. The dimensions of a pre-cracked specimen using for the finite element simulation [16].

$$
\begin{aligned}
& \boldsymbol{M}=\int_{V}^{\rho} \rho \boldsymbol{\Phi}^{T} \boldsymbol{\Phi} d V \\
& \boldsymbol{K}=\int_{V}\left\{\boldsymbol{B}^{T}\left(\boldsymbol{D}^{v}-\boldsymbol{F}^{\prime}\right) \boldsymbol{B}+\boldsymbol{E}^{T} \boldsymbol{Q}^{\prime} E\right\} d V, \\
& \dot{\boldsymbol{f}}_{1}=\int_{V} \dot{T} \boldsymbol{B}^{\boldsymbol{T}} \boldsymbol{B}^{e} d V, \quad \dot{\boldsymbol{f}}_{2}=\int_{V} \Theta_{1} \boldsymbol{B}^{T} \boldsymbol{P} d V, \\
& \dot{\boldsymbol{f}}_{3}=\int_{V} \Delta v \dot{f}^{\alpha^{\prime}} \boldsymbol{B}^{T}(\boldsymbol{Q}-\boldsymbol{\sigma}) d V, \\
& \dot{\boldsymbol{f}}_{t}=\int_{S_{t}} \boldsymbol{\Phi}^{T} \dot{\boldsymbol{F}} d S, \quad \dot{\boldsymbol{f}}_{g}=\int_{V} \boldsymbol{\Phi}^{T} \dot{\boldsymbol{G}} d V
\end{aligned}
$$

where $D^{v}, D^{e}, \boldsymbol{F}^{\prime}, \boldsymbol{Q}^{\prime}, \boldsymbol{P}, \boldsymbol{Q}, \boldsymbol{B}^{e}$ are the matrices corresponding to $D_{i j k l}^{v}, D_{i j k l}^{e}, F_{i j k l}^{\prime}, \sigma_{m j}, P_{i j}, Q_{i j}, B_{i j}^{e}$ respectively. $\dot{\boldsymbol{d}}^{e}$ is the nodal displacement rate and $\boldsymbol{\Phi}$ is the shape function of an element for the displacement rate. $\boldsymbol{E}$ and $\boldsymbol{B}$ are corresponding transformation matrices. $\rho \dot{\boldsymbol{G}}$ and $\dot{\boldsymbol{F}}$ are the rates of the body and surface forces, respectively.

Additionally, the FE heat conduction equation can be derived as

$$
\begin{gathered}
\overline{\boldsymbol{K}}_{1} \dot{\boldsymbol{t}}+\overline{\boldsymbol{K}}_{2} \boldsymbol{t}=\overline{\boldsymbol{R}}+\overline{\boldsymbol{Q}}-\overline{\boldsymbol{L}} \\
\overline{\boldsymbol{K}}_{1}=\int_{V} \rho C \overline{\boldsymbol{\Phi}}^{T} \overline{\boldsymbol{\Phi}} d V, \\
\overline{\boldsymbol{K}}_{2}=\int_{V} \kappa^{t} \overline{\boldsymbol{B}}^{T} \overline{\boldsymbol{B}} d V, \quad \overline{\boldsymbol{R}}=\int_{V} \overline{\boldsymbol{\Phi}}^{T} \zeta \bar{\sigma} \dot{\bar{\varepsilon}}^{p} d V, \\
\overline{\boldsymbol{Q}}=\int_{S_{q}} \overline{\boldsymbol{\Phi}}^{T} \bar{Q} d S, \quad \overline{\boldsymbol{L}}=\int_{V} \overline{\boldsymbol{\Phi}}^{T} \rho l_{\alpha^{\prime}} \dot{f}^{\alpha^{\prime}} d V
\end{gathered}
$$

where $\boldsymbol{t}$ is the nodal temperature vector, $\dot{t}$ is the rate of the nodal temperature vector, and $\overline{\boldsymbol{\Phi}}$ is the shape function for a temperature field. By using the Houlbolt Method, the second derivative of nodal displacement rate in time at moment $t+\Delta t, \ddot{d}_{t+\Delta t}^{e}$ can be rewritten.

\section{Three-dimension finite element model}

Figure 1 shows the dimension of a pre-cracked specimen using for the finite element simulation. Because of symmetric deformation, a quarter of the whole of the specimen is simulated. Figure 2 shows the 3D FE model established for the pre-cracked specimen. A hexahedral quadratic element with twenty nodes and reduced integration are used. The size of the mesh becomes smaller exponentially with approaching to crack-tip. The nodal displacement rate at the symmetrical boundary condition and the supporting point are set to be zero. The 


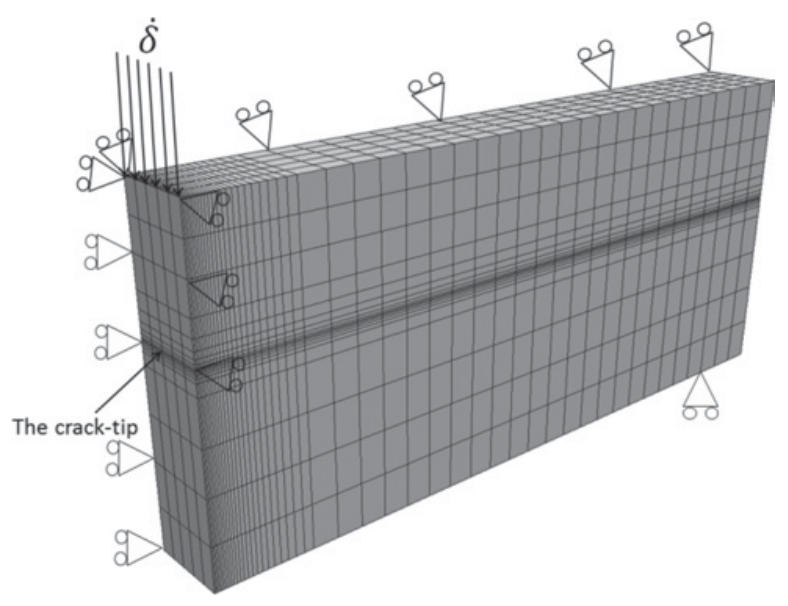

Figure 2. The 3D FE model for computational simulation.

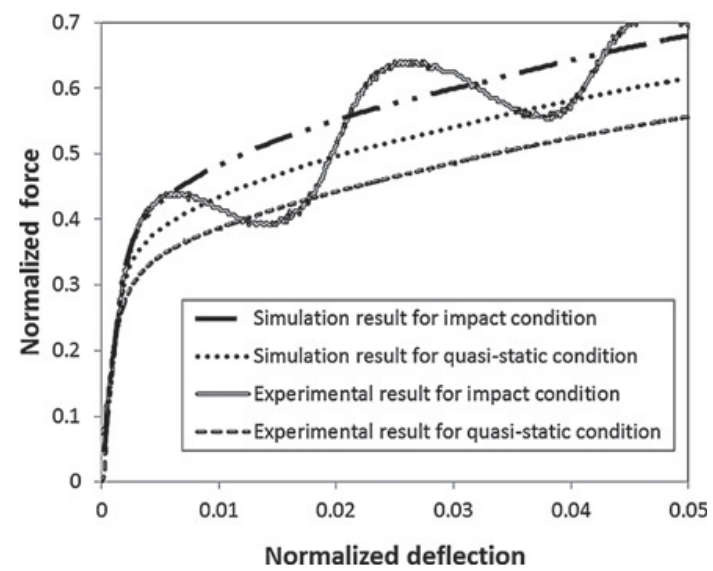

Figure 3. Normalized force vs normalized deflection under two cases of loading condition in experiment and simulation.

normalized deflection rate of $3.74 \times 10^{-2}$ and $10.44 / \mathrm{s}$ are set for simulation in the case of quasi-static and impact deformation, respectively. The environmental temperature is $298 \mathrm{~K}$.

\section{Results and discussion}

The value of force $P$ and deflection $\delta$ obtained from computational simulation is normalized as shown in the following equations.

$$
P_{n}=\frac{S}{4 Z \sigma_{y}} P, \quad \delta_{n}=\frac{4 W}{S^{2}} \delta
$$

where $\sigma_{y}$ is the initial yield stress at room temperature, $Z$ is the modulus of section, $S$ is the span lengths and $W$ is the width of the specimen.

In this study, energy-absorption is evaluated from normalized force-normalized deflection curve until the onset point of crack extension of specimen. Thus, the characteristic of normalized force-normalized deflection curve is examined. Figure 3 shows the relationship between normalized force and normalized deflection at normalized deflection rate of $3.74 \times 10^{-2}$ under quasi-static deformation and $10.44 / \mathrm{s}$ under impact loading deformation in experiment and simulation. Here,

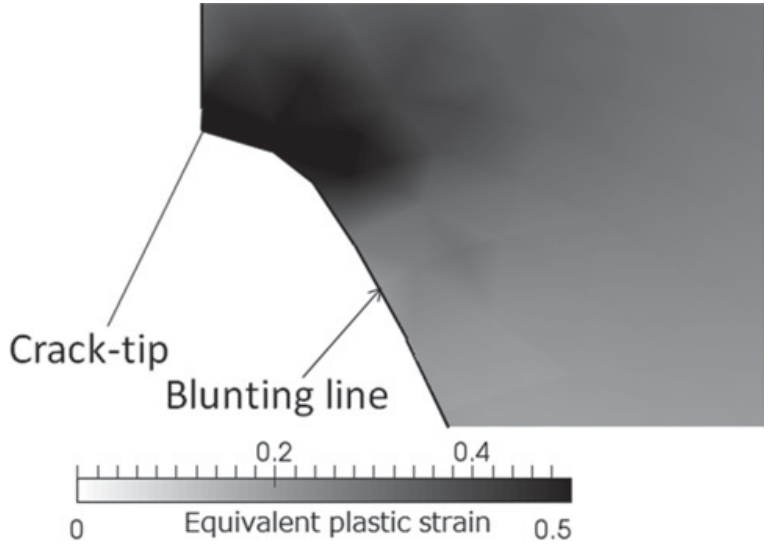

(a) Under quasi-static deformation

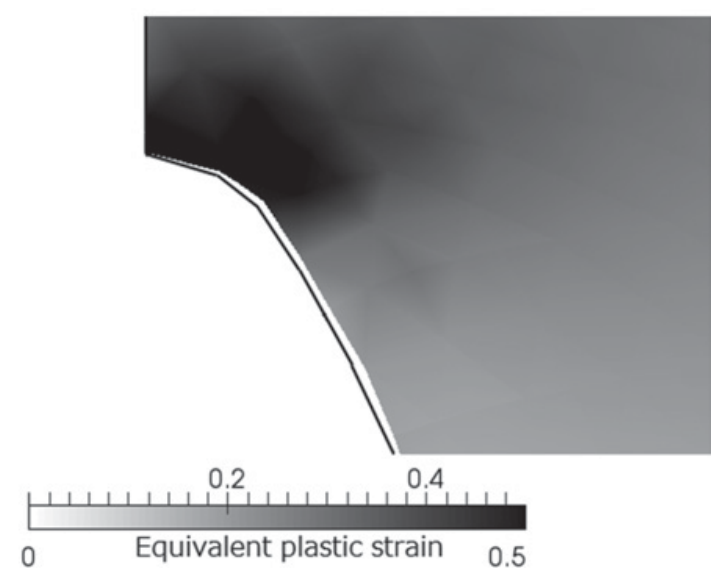

(b) Under impact deformation

Figure 4. The distribution of equivalent plastic strain under quasi-static and impact deformation at normalized deflection of 0.047 near crack-tip.

experimental results are obtained from Pham et al. [16]. A quite agreement between experimental and simulation results can be seen. Therefore, the validity of computation is confirmed. From this figure, it is clear that to obtain the same value of normalized deflection, external normalized force is required higher under impact condition than under quasi-static condition.

Next, the onset point of crack extension is determined through the blunting of crack tip. Figure 4 shows the distribution of equivalent plastic strain in the case of (a) quasi-static deformation, (b) impact deformation near crack-tip at normalized deflection of 0.047. It is clear that the crack has not extended yet. Here, the plastic deformation around the crack-tip leads to blunting the crack-tip. The blunting of crack-tip is larger under impact condition compare with under quasi-static condition at the same normalized deflection. As a result, the onset point of crack extension in case of impact deformation increases because the blunting of crack-tip induces resistance against crack initiation. From observations of Figs. 3 and 4, it can be said that energy-absorption in TRIP steel increases at a higher deflection rate.

Figure 5 shows the volume fraction of martensite with respect to normalized deflection. It is clear that the volume fraction of martensite under impact deformation 


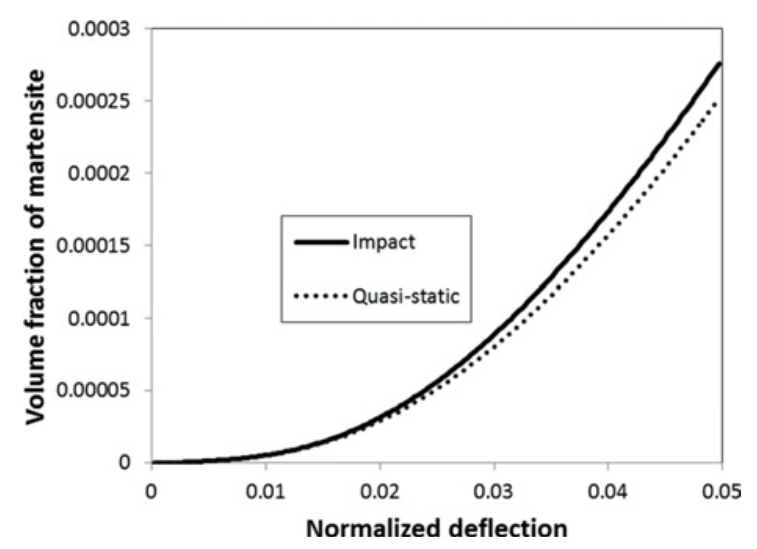

Figure 5. The relationship between volume fraction of martensite and normalized deflection under quasi-static and impact condition.

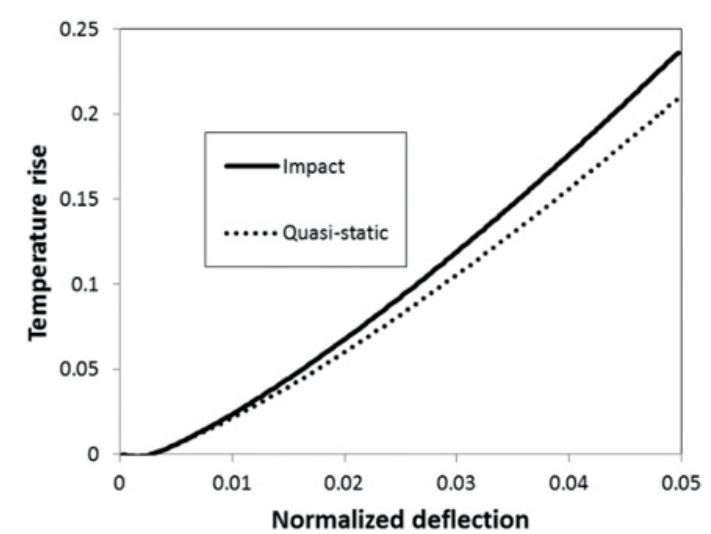

Figure 6. The relationship between temperature rise and normalized deflection under quasi-static and impact condition.

is higher than that under quasi-static deformation. A considerable value of volume fraction of martensite might be responsible for excellent energy-absorption characteristic in TRIP steel under impact loading as discussed above.

Figure 6 shows temperature rise with respect to normalized deflection for two cases of deformation. Here, temperature rise under impact deformation is higher than that under quasi-static deformation. This can be explained that at higher normalized deflection rate, adiabatic heating because of an inelastic irreversible work induces temperature rise. The distribution of temperature rise in specimen is shown in Fig. 7 in the case of (a) quasi-static deformation and (b) impact deformation at the normalized deflection of 0.047 . The distribution of temperature can be seen around the crack tip and loading point. Moreover, temperature rise is significantly higher under impact condition compare with under quasi-static condition. Although temperature increases considerably during impact deformation process, a high value of the volume fraction of martensite can be seen in Fig. 5. Thus, effect of other factors, except for temperature, needs to be considered.

Figure 8 shows the distribution of triaxiality for case of (a) quasi-static deformation and (b) impact deformation at the normalized deflection of 0.047 . In this figure, the

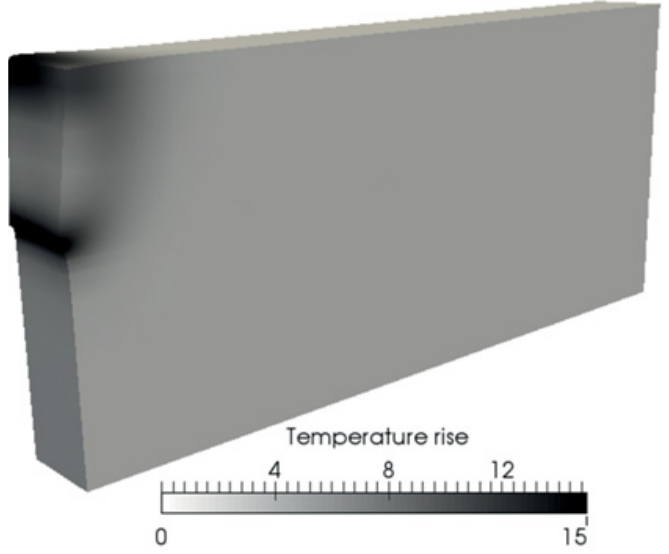

(a) Under quasi-static deformation

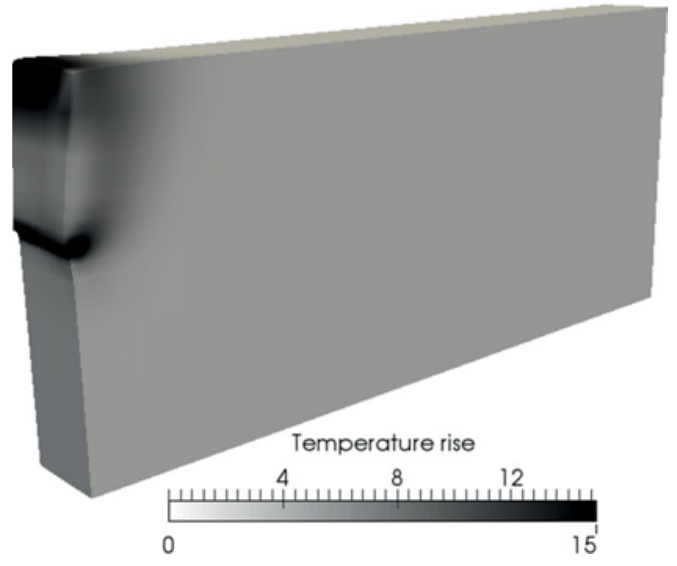

b) Under impact deformation

Figure 7. The distribution of temperature rise under quasi-static and impact deformation at normalized deflection of 0.047 .

triaxiality is distributed within three regions with value of $-0.33,0$ and 0.33 corresponding with compression, shear and tension, respectively. Compare with case of quasi-static condition, the compressive region in impact condition is slightly larger.

Next, Fig. 9 shows the distribution of volume fraction of martensite at the normalized deflection of 0.047 . Obviously, the volume fraction of martensite is distributed within compressive region, which is near the crack-tip and around the loading-point region. It can be said that compressive deformation creates more favorable situations for SIMT than tensile deformation because triaxiality is effective for shear band formation in the former case. Additionally, even though temperature rise is significantly high, the volume fraction of martensite is larger at a higher normalized deflection rate under impact loading. A larger compressive deformation as shown in Fig. 8 can be responsible for this result. Here, the stress triaxiality parameter $\Sigma$ strongly effects on SIMT rather than temperature. Moreover, at higher rate of deformation, the number of shear-band intersection is induced more as expressed in Eq. (1). As a result, volume fraction of martensite in impact condition is higher in quasi-static condition as observation in Fig. 5. 


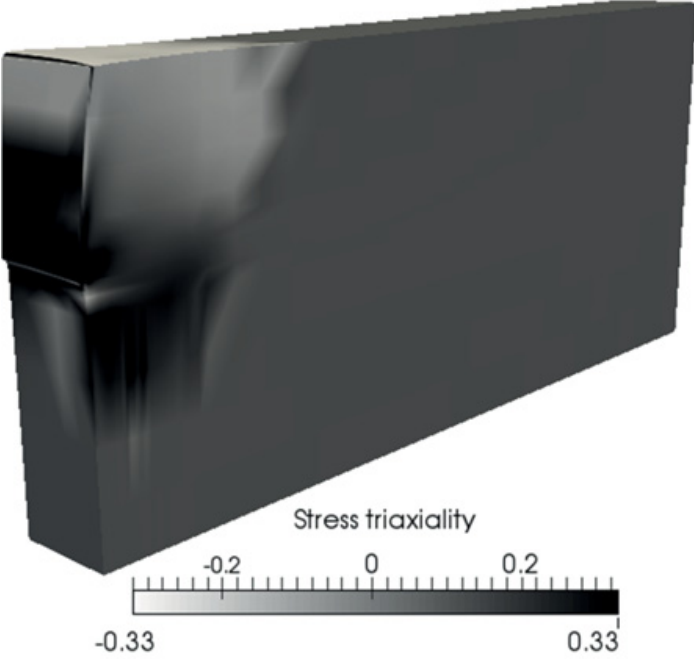

(a) Under quasi-static deformation

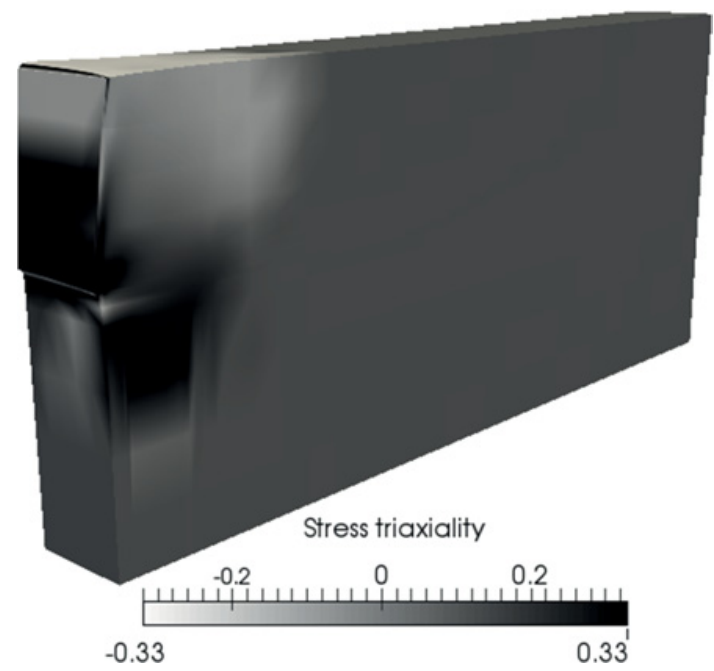

(b) Under impact deformation

Figure 8. The distribution of triaxiality under quasi-static and impact deformation at normalized deflection of 0.047 .

Next, distribution of abovementioned physical values near the crack tip is examined. Figure 10 shows the distribution of temperature rise for case of (a) quasistatic deformation and (b) impact deformation normalized deflection of 0.047 around the crack tip. A considerably high value of temperature rise can be seen here. Specially, under impact deformation, temperature increases higher than $M_{d}$ temperature. This might induce a softening thermal effect which leads a higher equivalent plastic strain near crack tip as shown in Fig. 4(b). Furthermore, it is expected that SIMT is suppressed under impact deformation around the crack tip because of high temperature rise.

Figure 11 shows the distribution of (a) triaxiality and (b) volume fraction of martensite near crack tip in case of impact condition at normalized deflection of 0.047. A compression region can be seen in the stretch zone of the crack tip. At the same time, volume fraction of martensite

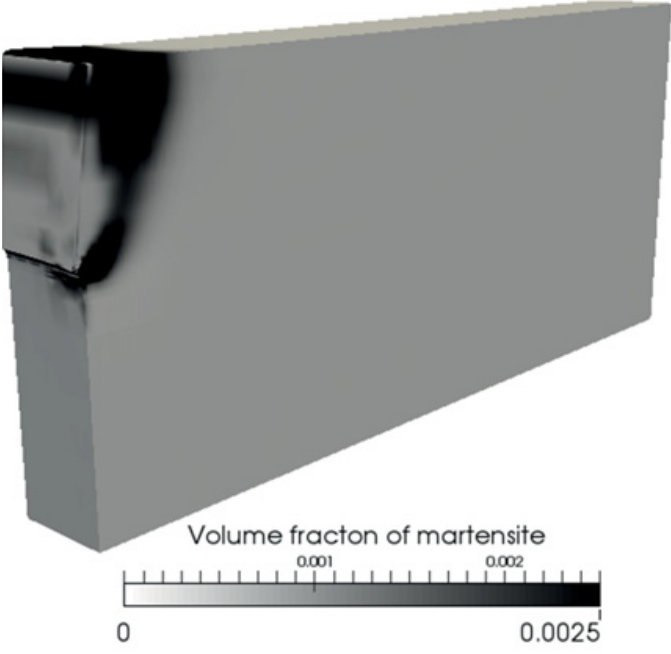

(a) Under quasi-static deformation

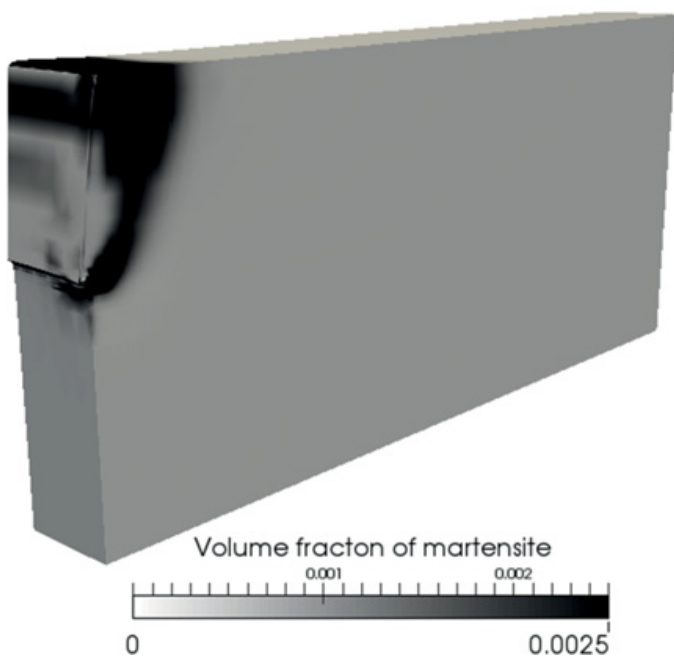

(b) Under impact deformation

Figure 9. The distribution of volume fraction of martensite under quasi-static and impact deformation at normalized deflection of 0.047 .

is distributed in this region even though temperature rise increases considerably. Clearly, compression deformation is more effective on SIMT than tension deformation. A considerable value of volume fraction of martensite might lead to delay the crack initiation, thus, energyabsorption of TRIP steel can be improved.

\section{Concluding remarks}

In this study, bending deformation behaviour of precracked specimen is investigated by $3 \mathrm{D}$ finite element simulation based on the transformation kinetics model. The concluding remarks are introduced as follows:

(1) To obtain same normalized deflection, external force is required higher under impact condition than 


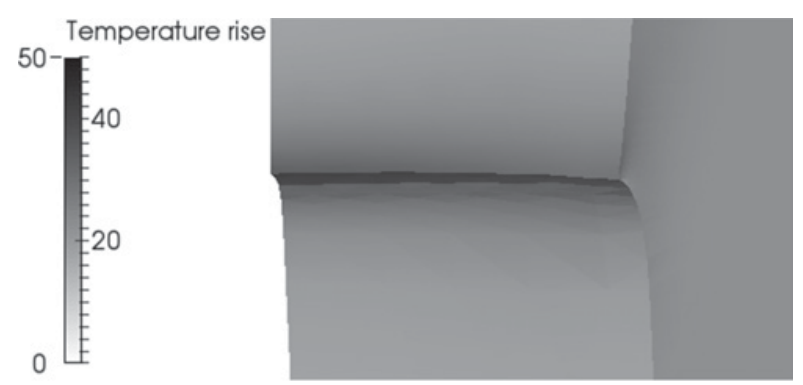

(a) Under quasi-static deformation

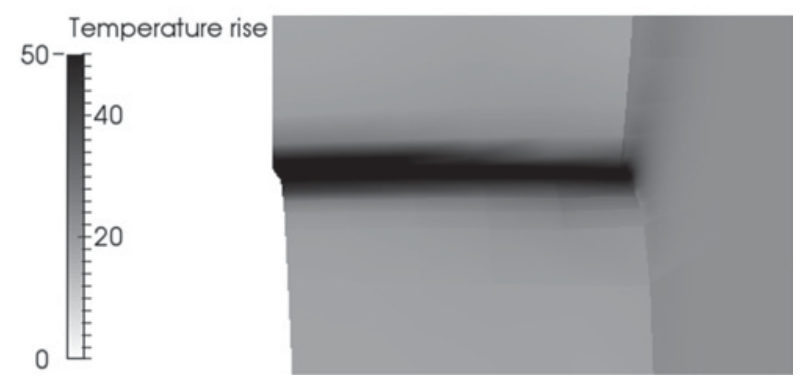

(b) Under impact deformation

Figure 10. The distribution of temperature rise under quasi-static and impact deformation at normalized deflection of 0.047 .

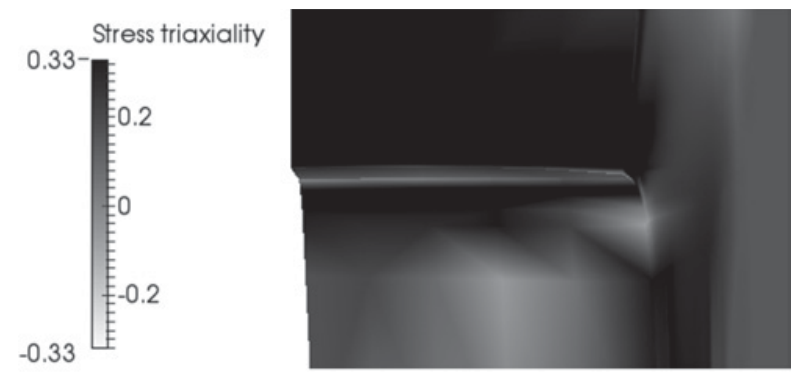

(a) The distribution of triaxiality

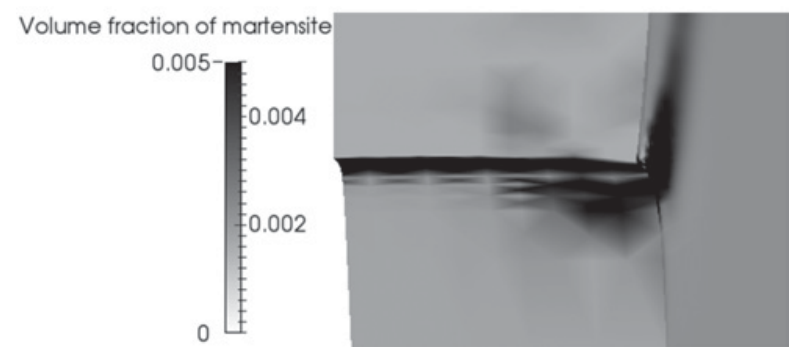

(b) The distribution of volume fraction of martensite

Figure 11. The distribution of triaxiality and volume fraction of martensite under impact deformation at normalized deflection of 0.047 .

under quasi-static condition. Moreover, blunting near crack tip under impact deformation is larger than that under quasi-static deformation. Therefore, it is considered that TRIP steel indicates better energy-absorption under impact condition than under quasi-static condition.

(2) The total volume fraction of martensite under impact deformation is higher than that under quasi-static deformation. This can be responsible for excellent energy-absorption capacity in TRIP steel at a higher deflection rate.

(3) Although temperature rise increases over $M_{d}$ around the crack tip under impact loading, volume fraction of martensite is distributed in compressive region. Here, triaxiality factor strongly effects on SIMT rather than temperature. Martensitic phase formed around the crack tip might lead to delay the crack initiation, thus, energy absorption of TRIP steel is improved.

We gratefully acknowledge financial supports from the $23^{\text {rd }}$ ISIJ Research Promotion Grant provided by the Iron and Steel Institute of Japan.

\section{References}

[1] M. Hori, JSAE Review 25, 3 (2004)

[2] IISI AUTOCO Report: www . worldautosteel.org

[3] I. Tamura, Met. Sci. 16, 245 (1982)

[4] T. Iwamoto, T. Sawa, M. Cherkaoui, Int. J. Mod. Phys. B 22, 5985 (2008)

[5] M.R. Berrahmoune, S. Berveiller, K. Inal, A. Moulin, E. Patoor, Mater. Sci. Eng. A 378, 304 (2004)

[6] F.D. Fischer, G. Reisner, E. Werner, K. Tanaka, G. Cailletaud, T. Antretter, Int. J. Plasticity 16, 723 (2000)

[7] S. Curtze, V.T. Kuokkala, M. Hokka, P. Peura, Mater. Sci. Eng. A 507, 124 (2009)

[8] J.R. Rice, J. Appl. Mech. 35, 379 (1968)

[9] ASTM E813-81, Annual Book of ASTM Standards, $810(1981)$

[10] G.B. Olson, M. Cohen, Metall. Trans. A 6, 791 (1975)

[11] R.G. Stringfellow, D.M. Parks, G.B. Olson, Acta Metall. Mater. 40, 1703 (1992)

[12] Y. Tomita, T. Iwamoto, Int. J. Mech. Sci. 37, 1295 (1995)

[13] T. Iwamoto, T. Tsuta, Int. J. Plasticity 16, 791 (2000)

[14] T. Iwamoto, T. Tsuta, Y. Tomita, Int. J. Mech. Sci. 40, 173 (1998)

[15] T. Iwamoto, T. Tsuta, Int. J. Plasticity 18, 1583 (2002)

[16] H.T. Pham, L. Shi, T. Iwamoto, Key. Mat. Eng. 626, 340 (2014) 